\title{
Clarification of Sugar Cane Juice (Saccha- rum officinarum) Through the Use of National Cocoa Muclago (Theobroma cacao L)
}

\section{Clarificación de Jugo de Caña de Azúcar (Saccharum officinarum) Mediante el Uso de Mucílago de Cáscara de Cacao Nacional (Theobroma cacao L)}

VII International Congress of Science, Technology, Entrepreneurship and Innovation (SECTEI 2020)

Corresponding Author:

A. Fernández

afernandez@uteq.edu.ec

Published: 26 August 2021

Production and Hosting by Knowledge E

(c) A. Fernández et al. This article is distributed under the terms of the Creative Commons Attribution License, which permits unrestricted use and redistribution provided that the original author and source are credited.

\author{
A. Fernández, D. Tuarez, C. Erazo, and E Torres \\ Universidad Técnica Estatal de Quevedo
}

\section{Abstract}

The investigation was carried out at 980 m.s.n. in the San Ramón compound of the El Corazón Parish, Pangua canton, Cotopaxi province, Ecuador. The variables to be evaluated in the sugar cane juice clarification process were the viscosity $(20,25$ and $30 \mathrm{cp})$ and the concentration of Ragnar variety cocoa shell mucilage $(0.75,1.5$, and $2.25 \%)$, to determine the spectral color and saturation in the sugarcane juice. 10 liters ofjuice were used in each experimental unit, taking the clarified syrup samples in amber bottles and refrigerating them until further analysis on the colorimeter was conducted. For the statistical evaluation of the results of the saturation percentage, normality tests were performed according to the Shapiro-Wilk statistic; applying the Friedman and Holm test of multiple comparisons, allowed to identify the combination of a1b2 $(25 \mathrm{cp} * 2.25 \%)$ as the best juice clarification treatment, and, managing to establish the descriptive data, thus: spectral color at $576 \mathrm{~nm}$, saturation 46.91\%; standard deviation 2.75; median $46.36 \%$; variance 7.6 and a range of 5.43 .

Keywords: syrup, spectral, flocculant, cachaza.

\section{Resumen}

La investigación se realizó a 980 msnm en el recinto San Ramón de la Parroquia El Corazón, cantón Pangua, provincia Cotopaxi, Ecuador. Las variables a evaluar en el proceso de clarificación de jugo de caña de azúcar fueron la viscosidad (20, 25 y 30 cp) y la concentración de mucílago de cáscara de cacao variedad Ragnar (0,75\%, 1,5\% y 2,25\%), para determinar el color espectral y de saturación en el jugo de caña de azúcar. En cada unidad experimental se usó 10 litros de jugo, tomando las muestras de jarabe clarificado en botellas tipo ámbar y refrigerándolas hasta posterior análisis en el colorímetro. Para la evaluación estadística de los resultados del porcentaje de saturación, se realizaron pruebas de normalidad según el estadístico de Shapiro-Wilk; aplicando el test de Friedman y Holm de comparaciones múltiples, permitieron identificar como mejor tratamiento de clarificación de jugo a la combinación a1b2 (25 cp * 2,25\%), y, logrando establecer los datos descriptivos, así: color espectral en $576 \mathrm{~nm}$, saturación 46,91\%; desviación estándar 2,75; mediana 46,36\%; varianza 7,6 y un rango de 5,43.

Palabras Clave: jarabe, espectral, floculante, cachaza. 


\section{Introducción}

La caña de azúcar es una planta muy antigua que se cultiva en países tropicales y subtropicales [1]. En el cantón Pangua, Ecuador se cultiva caña de azúcar de las variedades Ragnar y POJ-2878 y, los jugos se destinan a la elaboración de panela, aguardiente, miel y alfeñiques [2]. La panela y derivados de la caña de azúcar (Saccharum officinarum) se produce esencialmente en instalaciones de montaña conocidas como trapiches [3]; los trapiches en Pangua son de tipo rural con sistemas de producción artesanal basados en conocimientos ancestrales tradicionales sobre todo en la producción de aguardientes anisados tipo pájaro azul, la cosecha de la caña de azúcar se realiza mediante entresaque o desguíe y en los procesos productivos no se controlan muchas variables teniendo productos de calidad no estandarizada [2].

La estandarización de los factores que afectan la calidad de la panela, se clasifican en agroecológicos y de procesamiento [4], entre los de proceso están: el pH, la velocidad de calentamiento y clarificación [5]. El residuo sólido producido después de la filtración del jugo de caña de azúcar se presenta en forma de lodo [6]. Los mucílagos vegetales son extraídos y estudiados por diversos autores y con distintos fines; así, [7] en el estudio de 'Nuevas tecnologías desarrolladas para el aprovechamiento de las cactáceas en la elaboración de alimentos. Componentes funcionales y propiedades antioxidantes', concluyeron que, el mucílago aislado es una mezcla compleja de polisacáridos, de los cuales, menos del $50 \%$ corresponden a polímeros semejantes a las pectinas.

Adicionalmente, mediante el método Bradford no detectaron contenido de proteínas en el mucílago. Por otro lado, [8] exponen que la extracción del mucílago con agitación mecánica, no tiene influencia en la clarificación de jugos, pero se necesita que éste sea debidamente homogeneizado. Sin embargo, la clarificación de los jugos es mejor para concentraciones altas de extractos en la mayoría de las plantas estudiadas. Entre otros factores, el grado de extracción de los jugos de caña de azúcar tiene influencia en la calidad de la panela (color), mieles y alfeñiques; porcentajes de 60 a 65\% se consideran aceptables. Si supera estos valores, pueden afectar la calidad de los jugos debido al aumento de pectinas, gomas, ceras, grasas, y otros que dificultan el proceso de clarificación y genera coloraciones indeseables en los jugos [9].

En nuestro país, la corteza de cáscara de cacao nacional no es aprovechada, pudiéndose utilizar para la extracción de mucilago útil en la clarificación de jugos de caña de azúcar encaminándose a la utilización de desechos de la agricultura en la agroindustria panelera, según lo propuso [10]. Los floculantes, ya sean de origen químico o natural se emplean para aumentar la aglomeración de los flóculos, incrementar la velocidad de sedimentación, compactación y reducción del volumen de cachaza; mejorando la claridad (turbiedad) del jugo clarificado [2]. Existe la alternativa de reemplazar los mucílagos vegetales por compuestos químicos que realizan la misma función, pero en 
las investigaciones realizadas hasta el momento por el Centro de Investigación para el Mejoramiento de la Panela CIMPA no han superado a los resultados obtenidos con los mucílagos naturales, y en algunos de los casos presentan residualidad. De los polímeros se han evaluado las poliacrilamidas aniónicas, catiónicas y no iónicas [9].

La clarificación de jugos de caña de azúcar consiste en coagular los no azúcares por calentamiento a temperaturas muy cercanas a la de ebullición mediante la adición de algún agente clarificador [10]. La clasificación de las partículas suspendidas o material suspendido, en su mayoría, consiste de fragmentos de celulosa (bagacillo), tierra, arena, arcilla, almidón, ceras, grasas y gomas [11]. En el contexto anterior, el estudio del efecto de mucílago de cáscara de cacao nacional en la clarificación de jugos de caña de azúcar de la variedad ragnar, sirvió, para la determinación de las variables adecuadas del proceso de clarificación; y, brindar una nueva alternativa de mucílago con propiedades clarificantes al sector cañicultor.

Todo esto, considerando que en la Parroquia Moraspungo se cultiva cacao (Theobroma cacao L) nacional, CCN-51, híbridos y otros; y que, la cáscara del cacao no recibe ningún uso.

El uso alternativo de soluciones mucilaginosas a partir de la cáscara de cacao nacional en procesos de clarificación de jugos de caña de azúcar de la variedad ragnar, abre posibilidades de investigación en una operación unitaria crítica de jugos destinados a varios derivados, como son: panela (ya sea en atados, bloques o granulada), jugo natural envasado, mieles, alfeñiques y otros, dándole continuidad a la utilización de clarificadores naturales para una alimentación más sana. Investigaciones realizadas en el proceso de clarificación de jugo de caña de azúcar, independientemente de la variedad, recomiendan utilizar mucílagos vegetales con viscosidades desde 6 hasta 24 ср y dosis entre 0,1 a $6 \%$, con temperaturas y formas variables de adición, algunos lo añaden al inicio del proceso de calentamiento, mientras que otros lo añaden cuando ha alcanzado alrededor de $\operatorname{los} 55^{\circ} \mathrm{C}$, de forma total o fraccionada.

El mucílago se debe añadir al jugo de caña alrededor de los $60^{\circ} \mathrm{C}$ y, permitir un tiempo de acción adecuado para garantizar la formación de una espesa capa de cachaza que luego puede retirarse con facilidad [12]. Mientras [8] señalaron que la solución de mucílago de origen vegetal se emplea cuando los jugos han alcanzado una temperatura superior a los $60^{\circ} \mathrm{C}$. En Colombia, en las pruebas comparativas realizadas, se observó que muchas veces en el uso de mucílagos vegetales la temperatura inicial del proceso fue de alrededor de $56^{\circ} \mathrm{C}$ [9]. Añadido el mucílago, el proceso de transferencia de calor en la paila clarificadora de jugo de caña de azúcar juega un rol importante en la clarificación de los jugos, en donde existe un efecto combinado de 'temperatura tiempo' que permite la formación de unos flóculos o capa sobrenadante de impurezas conocida con el nombre de cachaza, que una vez retirada, es usada para la alimentación de cerdos y el abonado del cultivo. 
Con este antecedente, [13] recomienda que la temperatura de los jugos en calentamiento, una vez añadido la solución de mucílago se debe elevar entre 1 y $1,5^{\circ} \mathrm{C}$ por min. Mientras [14] señalaron que, la velocidad de calentamiento debe ser mayor a $1^{\circ} \mathrm{C} / \mathrm{min}$ para la obtención resultados satisfactorios. [9] señalaron que, una vez que se agrega el mucílago, el calentamiento hasta que se forme la cachaza negra antes de retirarlo de los jugos de la caña de azúcar cerca de $75^{\circ} \mathrm{C}$, debe durar 10 min. Para mejores resultados, la velocidad de calentamiento debe ser mayor a $1,5^{\circ} \mathrm{C}$ por min. $\mathrm{El}$ $\mathrm{pH}$ óptimo de acción de la enzima invertasa es de 3,5-5,0 [15] por lo que los jugos de caña de azúcar a ser clarificados deben estar por sobre el valor de pH 5,2 con valores entre 5,7 a 5,85 que conducen a un buen proceso de clarificación usando mucílagos vegetales. La actividad de los mucílagos vegetales es eficiente entre valores de $\mathrm{pH}$ 5,2 a 6,2; siendo los adecuados los valores de 5,7 a 5,8.

Una clarificación eficiente de jugos de caña de azúcar con mucílago de balso se encuentra en un $\mathrm{pH} 5,7$ [13]. El pH de los jugos en el momento de agregar el aglutinante es de 5,8 [9]. [2] reportó que, en San Ramón de la jurisdicción del cantón Pangua, los mejores resultados clarificando jugo de caña de azúcar, para producir panela granulada fueron utilizando mucílago de balso, con un pH de 5,8 a 5,85. Cuando los valores de pH no son los adecuados, su regulación en los jugos de caña de azúcar para producir panela se realiza mediante el uso de cal [9].

Antiguamente, la clarificación se hacía con cal y calor, lo que se conoce como un proceso de defecación simple y se lleva a cabo antes de que el jugo se concentre por evaporación. La cal produce reacciones con las sustancias en el jugo, de diferentes características: algunas dan lugar a compuestos que quedan en suspensión, otras producen precipitados y también ocurren reacciones que forman compuestos solubles en el jugo [16]. Al término de los referentes bibliográficos, fue importante el estudio de la viscosidad y la concentración a usarse de mucílago de cáscara de cacao nacional en la clarificación de jugos de caña de azúcar de la variedad ragnar cultivada en mayor extensión en la jurisdicción del cantón Pangua.

Para su cumplimiento de lo anterior, se planteó el objetivo: Analizar el efecto de la viscosidad y concentración del mucílago de cascara de cacao nacional en la clarificación de jugo de caña de azúcar de la variedad ragnar, para establecimiento de los indicadores de clarificación. El problema fue formulado así: ¿Cuál es el efecto de la viscosidad del mucílago de cascara de cacao nacional en el color de saturación (clarificación) de jugo de caña de azúcar de la variedad ragnar en un cultivo del recinto San Ramón, parroquia El Corazón, cantón Pangua? Se contrastó la siguiente hipótesis nula (Ho): Al utilizar mucilago de la cascara de cacao (T. cacao L) nacional a distintas viscosidades y porcentajes en el proceso de clarificación de los jugos de caña de azúcar de la variedad ragnar no existen diferencias de color de saturación de los jugos en el grupo de tratamientos. 


\section{Metodología}

El cacao nacional fue cosechado de la finca de la Sra. Zoila Escobar, ubicado a 280 msnm en el recinto Las Juntas de la Parroquia Moraspungo, de cuya materia prima solo se usó las cáscaras para extraer el mucílago el mismo que fue congelado por 4 días, posteriormente llevado hasta el Recinto San Ramón y utilizado en la clarificación de los jugos de caña de azúcar.

El estudio de clarificación de los jugos de caña de azúcar de la variedad ragnar, se realizó en la propiedad del Sr. René Paucar a 980 m.s.n.m., en el Recinto San Ramón de la Parroquia El Corazón en la jurisdicción del cantón Pangua, ubicado al sur occidente de la provincia de Cotopaxi. El cantón Pangua se extiende desde los 059' de latitud sur, $1^{\circ} 13^{\prime}$ en la parte septentrional y desde los $78^{\circ} 57^{\prime}$ de longitud occidental hasta los $79^{\circ} 29^{\prime}$ en la zona oriental; de clima cálido en parte baja y templado en la zona alta; temperatura media anual de $20^{\circ} \mathrm{C}$ y precipitación anual de 600 a $2000 \mathrm{~mm}$. Tiene una población aproximada de 22289 habitantes, distribuidos en una extensión territorial de 714,9 km² que corresponden a 4 parroquias: El Corazón, Moraspungo, Ramón Campaña y Pinllopata.
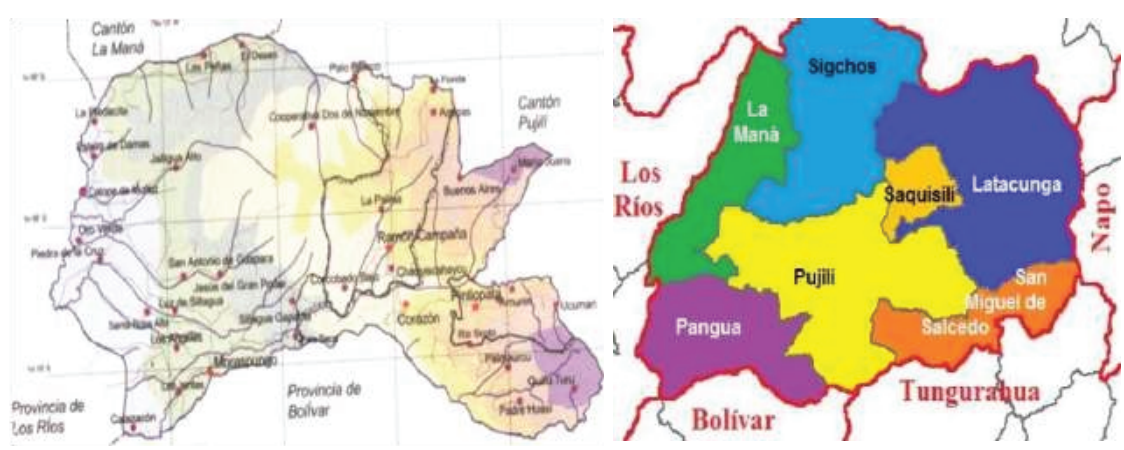

Figure 1

Mapa del Cantón Pangua en la Provincia de Cotopaxi [17].

\subsection{Extracción del mucílago de la cáscara de cacao nacional}

Para la extracción del mucílago de cáscara de cacao se empleó cáscara de cacao nacional, aguardiente de caña de azúcar de $60^{\circ} \mathrm{GL}$, metabisulfito, balanza analítica OHAUS de precisión $1 \mathrm{~g}$ y 0,0001g. viscosímetro de Ostwald, cronómetro, picnómetros, vasos de precipitación PYREX de 500 y 2000 mL.

La cosecha del cacao nacional de mazorcas maduras se realizó utilizando palancas de caña guadua con poladera bien afilada, una costumbre ancestral de los agricultores de Moraspungo y su entorno. Las mazorcas fueron lavadas para seguidamente cortar los polos o extremos y partir longitudinalmente con un machete de acero inoxidable, 
inmediatamente se separan las almendras de cacao y el maguey; al realizar el corte se selecciona la cáscara apta para el proceso desechando aquellas que presenten manchas oxidativas y/o ataque de monilla.

La cáscara de cacao nacional fue picada finamente, con un espesor entre 0,3-0,6 $\mathrm{mm}$ y en una balanza de precisión $0,1 \mathrm{~g}$. Pesada la masa requerida de cáscara y metabisulfito, también se midió el volumen de agua y de aguardiente, la mezcla fue la siguiente: cáscara de cacao nacional $25 \%$; agua $73,59 \%$; aguardiente de $60^{\circ} \mathrm{GL} 1,40 \%$ y metabisulfito de sodio 0,01\%. El agua debe estar a ebullición, a esta temperatura se añade el metabisulfito agitando la solución y seguido se agrega la cáscara de cacao nacional picada y el aguardiente. Al incorporar los ingredientes al agua hirviendo, desciende la temperatura, se deja hervir por 5 min con agitación leve.

Retirado del fuego, se enfría la mezcla y cuando ya está temperada la solución, se procede a estrujar los trozos de cáscara de cacao por un minuto cada vez, a intervalos de 5 a 6 min. Transcurridos aproximadamente 80 min contabilizados a partir de la inmersión de las cáscaras en el agua hirviendo, se procede a tamizar la solución.

Una vez tamizado el mucílago, se analizó la viscosidad con el viscosímetro de Ostwald, este equipo determinó el tiempo de caída del agua y de la solución mucilaginosa extraída, el tubo capilar se sumergió en agua a $25^{\circ} \mathrm{C} \pm 0,2^{\circ} \mathrm{C}$. Paralelamente se determinó la densidad de la solución mucilaginosa usando picnómetros. Finalmente, la viscosidad de la solución mucilaginosa de cacao nacional se calcula usando la ecuación (1).

La relación entre las viscosidades 1 y 2 de los dos líquidos cuyas densidades son $\delta_{1} y \delta_{2}$ es:

$$
\frac{\eta_{1}}{\eta_{2}}=\frac{\delta_{1} t_{1}}{\delta_{2} t_{2}}
$$

donde $\mathrm{t} 1$ y $\mathrm{t} 2$ son los tiempos de flujo.

La solución mucilaginosa de 30 o más de $30 \mathrm{cp}$ se acondicionó a 20, 25 y $30 \mathrm{cp}$ y se envasó en cantidades correspondientes a 0,75, 1,5 y 2,25\% considerando cada unidad experimental de $10 \mathrm{~L}$ de jugo de caña de azúcar.

\subsection{Preparación de la muestra de jugo de caña}

La fase experimental se desarrolló en los meses de septiembre y octubre; época seca en la jurisdicción del cantón Pangua, en donde la caña de azúcar presenta su mejor contenido de sólidos solubles.

La caña de azúcar se cosechó en la modalidad de entresaque o desguíe, pues en Pangua no se realiza el corte mediante zafra como es en los ingenios azucareros. Una vez cortada la caña de azúcar, inmediatamente fue transportada a la unidad de producción (trapiche) a través de acémilas. 
Los tallos de caña de azúcar fueron limpiados, retirando raicillas y musgos y sometidos a lavado para retirar tierra, arena y excrementos de pájaros y/o insectos presentes en los tallos.

Debido al espesor de los tallos, fue pertinente cortarlos en dos y/o hasta en cuatro partes de manera longitudinal. Esta operación permitió observar cañas que habían sufrido ataques de insectos y que presentaron índices de sobre maduración, las que fueron eliminadas.

Para extraer el jugo se utilizó un molino mecánico, siguiendo el procedimiento que mantienen los cañicultores de la zona, el tiempo estimado de la extracción no supero 10 min, variable importante dentro de esta operación que evita la fermentación del jugo.

El jugo extraído de la molienda, se sometió a un prelimpiador colocado a la salida del molino, antes de llegar a la tina recibidora. Inmediatamente se midió el volumen de 10 litros de jugo y se añadió metabisulfito, en una relación de 125 ppm, para contrarrestar la fermentación del jugo.

Se midió el $\mathrm{pH}$ al jugo extraído, presentando un valor homogéneo en todos los tratamientos $(5,77)$, no siendo necesario añadir cal. Para este fin se utilizó un pH-metro digital ECO TESTR pH2. El valor promedio de los sólidos solubles, medido con un brixómetro de marca OECHSLE de 0-35\% fue: para patrón y tratamientos con mucilago

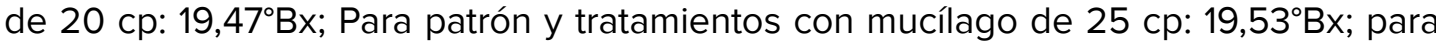
patrón y tratamientos con mucílago de $30 \mathrm{cp}: 19,65^{\circ} \mathrm{Bx}$.

\subsection{Clarificación del jugo de caña de azúcar}

Los tratamientos del jugo de caña, fueron calentados hasta alcanzar $56^{\circ} \mathrm{C} \pm 2^{\circ} \mathrm{C}$, previo a la adición del mucílago. La temperatura se midió con un termómetro bimetálico marca MULTI-THERMOMETER LCD DIGITAL. Una vez que el jugo alcanzó la temperatura de $56^{\circ} \mathrm{C} \pm 2{ }^{\circ} \mathrm{C}$, se procedió a añadir el $75 \%$ de mucílago (previamente descongelado) de cáscara de cacao nacional según consta en el diseño experimental propuesto en esta investigación.

Al añadirse el mucílago se agitó el jugo de caña de azúcar por espacio de 30 segundos, con el fin que el floculante natural se homogenice en el sistema. Añadido el mucílago de cáscara de cacao nacional, se controló que la temperatura de los jugos suba gradualmente a una razón de $1,5 \pm 0,2^{\circ} \mathrm{C} / \mathrm{min}$. En todos los tratamientos, para permitir la acción del floculante natural sobre las impurezas que tiene el jugo de caña de azúcar, y comience la formación de la cachaza negra sobrenadante. Con la gradiente de temperatura controlada, y una vez que el jugo alcanzó $75^{\circ} \mathrm{C} \pm 2^{\circ} \mathrm{C}$ en aproximadamente 12 min, se formó una cachaza negra bastante espesa, ésta se separó con un tamiz casero. 
Retirado la cachaza negra del jugo de caña se añade el 25\% de mucílago de cáscara de cacao nacional restante, con el fin de que salga la cachaza más liviana, que se torna blanquecina y que se dificulta el retiro, esto debido a que, se observó que el movimiento de los jugos por el proceso de transferencia de calor por convección se acelera sobre los $85^{\circ} \mathrm{C}$ y se rompen los flóculos formados.

Transcurrido 5 min, se tomó muestras, envasadas en botellas color ámbar. Las muestras de jugo envasadas fueron refrigeradas, para posteriormente trasladarlas en empaques térmicos al laboratorio FICAYA de la Universidad Técnica del Norte, en la ciudad de Ibarra, donde se determinó el color espectral y de saturación en el colorímetro en un espacio de tiempo de $48 \mathrm{hr}$ después del tratamiento de clarificación.

\subsection{Análisis colorimétrico}

La determinación del color espectral y saturación del jugo clarificado de caña de azúcar, se realizó mediante un Espectrofotómetro ANALYTIK JENA, modelo SPECORD 250 plus.

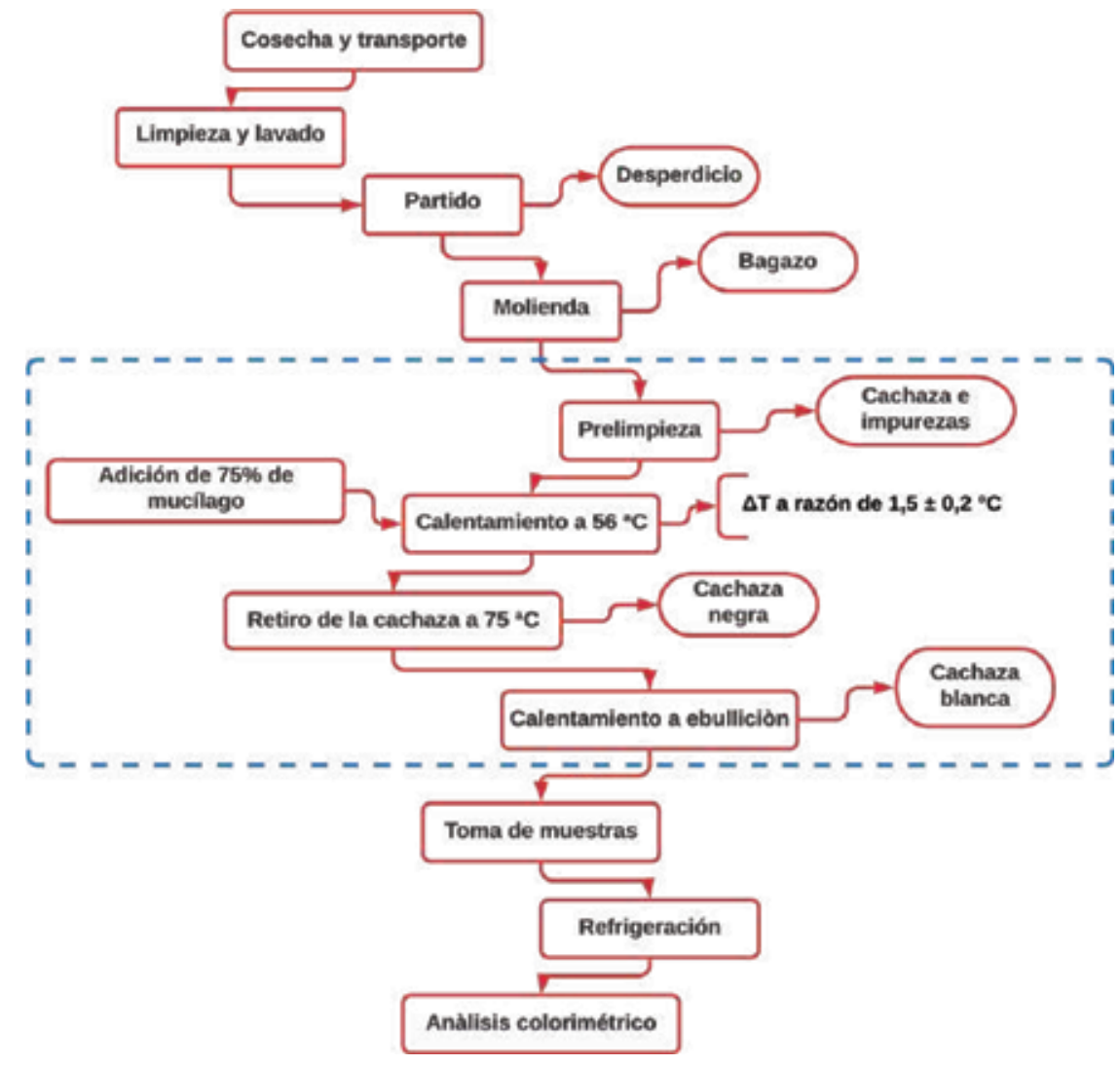

Figure 2

Diagrama de bloques mostrando corrientes de entrada y salida del proceso de clarificación del jugo de caña de azúcar de la variedad 201 ragnar usando mucílago de cáscara de cacao nacional. 


\section{Resultados y Discusión}

\subsection{Extracción del mucílago}

En la extracción del mucílago, los datos que se obtuvieron de viscosidad expresados en centipoise (cp.) al trabajar 205 con cáscara finamente picada de cacao nacional maduro se desprendió el mucílago a la viscosidad requerida en esta 206 investigación en el menor tiempo; es decir que, en alrededor de 60 min se obtuvo una viscosidad de $25,51 \mathrm{cp}$. y 207 al cabo de 80 min una viscosidad de 30,93 cp.

\subsection{Clarificación del jugo de caña}

Los resultados de la saturación expresados en porcentaje obtenidos en el espectrofotómetro ANALYTIK JENA, Modelo SPECORD 250 plus (colorímetro), de las muestras de jugos de caña de azúcar de la variedad ragnar 211 clarificados con mucílago de cáscara de cacao nacional en tres viscosidades y tres concentraciones fueron reportados 212 en la Tabla 1.

\section{Table 1}

Saturación de los jugos de caña de azúcar de la variedad ragnar clarificados con mucílago de cáscara de cacao nacional.

\begin{tabular}{|c|c|c|c|c|c|}
\hline \multicolumn{6}{|c|}{ Combinación de Réplicas } \\
\hline No. & Símbolo & tratamientos & R1 (\%) & R2 (\%) & R3 (\%) \\
\hline 1 & & Patrón & 67,83 & 63,91 & 64,41 \\
\hline 2 & aobo & 20 ср. $\wedge 0,75 \%$ & 57,13 & 58,02 & 59,20 \\
\hline 3 & aob1 & 20 ср. $\wedge 1,5 \%$ & 57,61 & 51,57 & 57,31 \\
\hline 4 & aob2 & 20 ср. $\wedge 2,25 \%$ & 49,05 & 49,90 & 51,70 \\
\hline 5 & a1bo & 25 ср. $\wedge 0,75 \%$ & 55,85 & 55,06 & 57,03 \\
\hline 6 & a1b1 & 25 ср. $\wedge 1,50 \%$ & 51,00 & 50,76 & 51,40 \\
\hline 7 & $\mathrm{a} 1 \mathrm{~b} 2$ & 25 ср. $\wedge 2,25 \%$ & 44,47 & 49,89 & 46,36 \\
\hline 8 & a2bo & 30 ср. $\wedge 0,75 \%$ & 54,89 & 45,30 & 52,30 \\
\hline 9 & $\mathrm{a} 2 \mathrm{~b} 1$ & 30 ср. $\wedge 1,50 \%$ & 57,45 & 56,30 & 57,95 \\
\hline 10 & $\mathrm{a} 2 \mathrm{~b} 2$ & 30 ср. $\wedge 2,25 \%$ & 47,26 & 52,14 & 50,93 \\
\hline
\end{tabular}

$\mathrm{CP}$ : centipoise.

Para la interpretación de los resultados se parte de un análisis estadístico de prueba de normalidad de los valores de 217 saturación reportados en la Tabla 1 que conduce a construir la Tabla 2. Aquí, se contrasta la hipótesis nula HO: Los 218 datos de color de saturación de los jugos de caña de azúcar de la variedad ragnar clarificados con mucílago de cáscara 219 de cacao nacional en tres viscosidades y tres concentraciones se distribuyen de forma normal. 
Table 2

Pruebas de normalidad.

\begin{tabular}{|l|l|l|}
\hline & $\begin{array}{l}\text { Kolmogorov- } \\
\text { Smirnova } \\
\text { Estadístico }\end{array}$ & GI \\
\hline Patrón & 0,34 & 3 \\
\hline aOb0 & 0,20 & 3 \\
\hline a0b1 & 0,37 & 3 \\
\hline a0b2 & 0,26 & 3 \\
\hline a1b0 & 0,22 & 3 \\
\hline a1b1 & 0,24 & 3 \\
\hline a1b2 & 0,25 & 3 \\
\hline a2b0 & 0,28 & 3 \\
\hline a2b1 & 0,27 & 3 \\
\hline a2b2 & 0,29 & 3 \\
\hline
\end{tabular}

\begin{tabular}{l|l|l|l|} 
& & \multicolumn{3}{|c|}{ Shapiro- Wilk } \\
\hline Sig. & Estadístico & GI & Sig. \\
\hline 0,84 & 3 & 0,22 \\
\hline 0,99 & 3 & 0,85 \\
\hline 0,78 & 3 & 0,08 \\
\hline 0,96 & 3 & 0,61 \\
\hline 0,99 & 3 & 0,78 \\
\hline 0,97 & 3 & 0,69 \\
\hline 0,97 & 3 & 0,67 \\
0,93 & 3 & 0,51 \\
0,95 & 3 & 0,57 \\
0,92 & 3 & 0,46 \\
\hline
\end{tabular}

${ }^{a}$ Corrección de significación de Lilliefors

Se aplicó un conjunto de test no paramétricos para identificar el mejor tratamiento.

A continuación, se evidenció el resultado del test de Friedman a partir de la hipótesis planteada en esta investigación. 225 Además, se mostraron los valores de ranking de cada uno de los tratamientos.

Table 3

Ranking de valores de los tratamientos, al aplicar el test de Friedman.

\begin{tabular}{ll} 
Algorithm & Ranking \\
\hline Patrón & 10,00 \\
aOb0 & 8,33 \\
aOb1 & 7,00 \\
aOb2 & 3,33 \\
a1b0 & 6,33 \\
a1b1 & 3,66 \\
a1b2 & 1,33 \\
a2b0 & 3,66 \\
a2b1 & 8,00 \\
a2b2 & 3,33 \\
& P-valor computado por Friedman Test: 0,005
\end{tabular}

Se apreció que el valor computado por el test de Friedman de 0,005 es menor que $\alpha=0,05$ por lo que, se rechazó la 228 hipótesis nula HO que se planteó. 
En la Tabla 3 se observó que, la combinación de tratamientos que mejor valor medio se obtuvo con el tratamiento 230 a1b2 ( $25 \mathrm{cp} * 2,25 \%$ ), esto es viscosidad de $25 \mathrm{cp}$ y una concentración del 2,25\% en relación al volumen de jugo a ser clarificado.

En la Tabla 4 se presentó los resultados de comparaciones múltiples del test de Holm [18], tomando como tratamiento 233 de control la combinación a1b2 elegido según el test de Friedman [19]. La característica del test Holm es que ordena 234 los valores de forma descendente en cuanto a la magnitud de la diferencia.

\section{Table 4}

Resultados del test de Holm $(\alpha=0,05)$.

\begin{tabular}{ll|l|l|l}
8 & aOb0 & 2,83 & 0,004 & 0,006 \\
\hline 7 & a2b1 & 2,69 & 0,007 & 0,007 \\
\hline 6 & aOb1 & 2,29 & 0,021 & 0,008 \\
\hline 5 & a1b0 & 2,02 & 0,043 & 0,011 \\
\hline 4 & a2b0 & 0,94 & 0,345 & 0,012 \\
\hline 3 & a1b1 & 0,94 & 0,345 & 0,016 \\
\hline 2 & aOb2 & 0,80 & 0,418 & 0,025 \\
\hline 1 & a2b2 & 0,80 & 0,418 & 0,050
\end{tabular}

Al analizar la Tabla 4, respecto al tratamiento a1b2 (25 cp * 2,25\%) se pudo apreciar las columnas p y Holm, y, para el caso patrón (jugo de caña de azúcar tratado en condiciones iguales, pero sin adición de mucílago ni metabisulfito de sodio), aOb0 (20 $c p * 0,75 \%$ ), y a2b1 (30 cp *1,5\%) presentan valor $p<$ valor Holm, esto ratifica que los resultados de clarificación de jugos de caña de azúcar del tratamiento a1b2 son significativamente mejores.

El tratamiento a1b2 (25 cp * 2,25\%) no fue significativamente mejor que los obtenidos por los otros tratamientos: $a 0 b 1, a 1 b 0, a 2 b 0, a 1 b 1, a 0 b 2$ y a2b2; aunque se pudo observar que, el valor medio de a1b2 es menor que los tratamientos, no se pudo corroborar que la diferencia sea significativa.

Una particularidad que se pudo observar en el test de Holm, es que, los valores medios menores de comparación se dan con la combinación b2, es decir, los tratamientos que une la viscosidad con 2,25\% de mucílago añadido a los jugos de caña de azúcar a tratarse, arrojaron mejores resultados en el color de saturación.

La adición de mucílago de balso de 24 cp. y 1,5\% del volumen de jugo a tratarse, permitió la obtención de una cantidad satisfactoria de cachaza sobrenadante y no afectó la turbidez de los jugos de caña de azúcar clarificados [13]. El mucílago de balso con viscosidad de $6 \mathrm{cp}$. se debe adicionar $2,5 \%$, en referencia al volumen de jugo de caña de azúcar a clarificar para obtener resultados satisfactorios [9].

En los procesos de clarificación, las soluciones floculantes se usaron en dosis que varían de $0,1 \%$ a $4 \%(p / v)$ [20]. En varios lugares de Colombia se utilizaron entre 15 y 
30 litros de solución mucilaginosa por cada 500 litros de jugo de caña de azúcar a clarificar [8]. Es decir, la dosis de mucílago varía entre 3 y $6 \%$.

Lo anterior demostró que, la dosificación de soluciones mucilaginosas o floculantes naturales varía entre 0,1 y 6\% según lo indicado por los investigadores de esta área. Así, en esta investigación, luego del análisis estadístico de los resultados de saturación de los jugos clarificados se estableció que la viscosidad del mucílago de cáscara de cacao nacional y dosis en relación al volumen de jugo de caña de azúcar de la variedad ragnar es de $25 \mathrm{cp}$. y $2,25 \%$ respectivamente.

\subsection{Análisis colorimétrico}

El color espectral y de saturación de los jugos clarificados de caña de azúcar, usando mucílagos de cáscara de cacao nacional o de otro floculante natural no existe referencias que permitan realizar un análisis respetuoso y ponderado de este parámetro de calidad.

Los datos determinados para el patrón de jugo usado para comparar el mejor tratamiento, fueron los siguientes: color espectral 576 nm, y de saturación 63,91\%; mientras que, para el mejor tratamiento $\left(a_{1} b_{2}\right)$ el color espectral también fue de $576 \mathrm{~nm}$ y de saturación $46,91 \%$.

Con lo anterior, se calculó el porcentaje de aclaración de los jugos de caña de azúcar clarificados usando mucílago de cáscara de cacao nacional, mediante la siguiente ecuación (2).

$$
\% A J C A=\left(\frac{C E_{p}-C E_{t}}{C E_{p}}\right) * 100
$$

donde:

\% AJC: Porcentaje de aclaración de los jugos de caña de azúcar;

CEp: Color espectral del patrón; y

CEt: Color espectral del tratamiento.

$$
\begin{gathered}
\% A J C A=\left(\frac{63,91 \%-46,91 \%}{63,91 \%}\right) * 100 \\
\% A J C A=26,61 \%
\end{gathered}
$$

El resultado demostró la existencia de un efecto positivo del uso de mucílago de cáscara de cacao nacional en una viscosidad de $25 \mathrm{cp}$ y una dosis de 2,25\% en los jugos de caña de azúcar clarificados ya que se aclararon en un $26,61 \%$.

Así, se generó base para posteriores investigaciones en esta área, teniendo en cuenta que el color es un parámetro que define la calidad de los jugos y conduce a la obtención un color amarillo brillante de la panela, ya sea en atados o granulada. 


\section{Conclusiones}

Se observó que los valores de significancia de Shapiro-Wilk son mayores que $\alpha=0,05$ en consecuencia, la hipótesis nula $(\mathrm{HO})$ es aceptada, demostrando que los datos se distribuyen de forma normal.

La clarificación utilizando mucilago de cacao ( $T$. cacao $L$ ) con viscosidad de $25 \mathrm{cp}$. y $2,25 \%$ (tratamiento a1b2) en relación al volumen de jugo a tratar debe ser utilizado en procesos de clarificación de jugos de caña de azúcar (Saccharum officinarum) de la variedad ragnar; debido a que, el estudio realizado arrojo que su valor medio es mejor en comparación los demás tratamientos y significativamente supero al patrón (jugo de caña sin adición de mucílago), aOb0 (20 cp. * 0,75\%), y a2b1 (30 cp. ${ }^{*}$ 1,5\%).

Los jugos de caña de azúcar de la variedad ragnar cultivados en el cantón Pangua a una altura promedio de 980 m.s.n.m. y clarificados con mucílago de cáscara de cacao nacional de viscosidad $25 \mathrm{cp}$. y concentración 2,25\%, tienen un color espectral $576 \mathrm{~nm}$ y de saturación 46,91\%; la muestra de jugo patrón presentó un color espectral 576 nm y de saturación $63,91 \%$, arrojando como efecto una aclaración del $26,61 \%$; mejorando significativamente la calidad del jugo de caña de azúcar.

\section{Conflicto de Intereses}

De acuerdo a los parámetros solicitados para efectuar la publicación del artículo, se afirma que los resultados que produjo esta investigación no tienen ningún conflicto de interés con los autores, ni con la institución donde se realizó.

\section{References}

[1] Fito J, Tefera N, Van Hulle SWH. Sugarcane biorefineries wastewater: bioremediation technologies for environmental sustainability. Chem Biol Technol Agric [Internet]. 2019;6(1):1-13. Available from: https://doi.org/10.1186/s40538-019-0144-5

[2] Fernández Escobar A. Efecto de la viscosidad del mucílago de cáscara de cacao (Theobroma cacao I) nacional en la clarificación de jugo de caña de azúcar (saccharum officinarum) de la variedad ragnar. Universidad Agraria del Ecuador; 2016.

[3] Díaz A, Iglesias CE. Bases teóricas para la fundamentación del proceso de extracción de jugo de caña de azúcar para la producción de panela Theoretical bases of the sugar cane juice extraction process for the panela production. Vol. 21, Revista Ciencias Técnicas Agropecuarias. 2012.

[4] Mujica, María; Guerra, Marisa; Soto N. Efecto de la variedad, lavado de la caña y temperatura de punteo sobre la calidad de la panela granulada. Interciencia 
[Internet]. 2008 [cited 2020 Jan 3];33(8):598-603. Available from: http://www. redalyc.org/articulo.oa?id=33933808

[5] Demera Lucas, Francisco; Almeida Vera, Alex; Moreira Palacios, Carlos; Zambrano Velásquez, Lenin; Loor Cusme, Rosanna; Cedeño Alcívar D. Clarificación del jugo de caña de azúcar (saccharum officinarum I.) mediante el empleo de mucílagos naturales. Aliment Hoy. 2015;23(36):157-64.

[6] Kumar V, Chopra A K. Effects of sugarcane pressmud on agronomical characteristics of hybrid cultivar of eggplant (Solanum melongena L.) under field conditions. Int J Recycl Org Waste Agric. 2016;5:149-62.

[7] Nazareno M, Padrón C. Nuevas tecnologías desarrolladas para el aprovechamiento de las cactáceas en la elaboración de alimentos. Componentes funcionales y propiedades antioxidantes. Rev Venez Cienc y Tecnol Aliment. 2011;2(1):202-38.

[8] Quezada Moreno, Walter; Gallardo Aguilar I. Obtención de extractos de plantas mucilaginosas para la clarificación de jugos de caña Obtainment of Mucilaginous Plant Extrax for Clarification of Cane Juice. Tecnol Química. 2014;XXXIV(2):91-8.

[9] Durán Castro N, Gil Zapata N, García H. Manual de elaboración de panela y otros derivados de la caña. In: Convenio de investigacion y divulgacion para el mejoramiento de la industria panelera ICA - HOLANDA. Barbosa, (S.S) Julio 1992 Colombia; 1992. p. 312.

[10] Perez P. Mucílago pulverizado obtenido a partir de la cáscara de cacao, una alternativa en la clarificación de jugos en la industria panelera. Universidad Nacional de Colombia sede Manizales; 2004.

[11] Avalos Ludeña J, Ponte Ramirez R. Efecto del pH y temperatura en la clarificación del jugo de caña de azúcar (Saccharum Officinarum) por carbonatación. Universidad Nacional del Santa; 2019.

[12] Chudt A. Avances en el cultivo de la caña y elaboracion de la panela. In: Convenio ICA - Holanda de Investigacion y divulgacion para el mejoramiento de la industria panelera en Colombia. Barbosa, Colombia; 1998. p. 9.

[13] Paz Riofrío H. Tratamiento del jugo de caña para la elaboración de panela [Internet]. Universidad Politecnica Nacional; 1992 [cited 2020 Jan 4]. Available from: https://biblioteca.epn.edu.ec/cgi-bin/koha/opacdetail.pl?biblionumber= 4321\&query_desc=su\{\%\}3A\{\%\}22PANELA\{\%\}22andsu-to\{\%\}3ACANADEAZUCAR

[14] Velasco Caicedo, Diana; Rivera SI. Estandarización de una fórmula de aglutinante natural exraido de la planta cadillo (Triumfetta láppulal) para emplearse como clarificante en la producción de canela. Universidad de San Buenaventura; 2011.

[15] Andjelković U, Pićurić S, Vujčić Z. Purification and characterisation of Saccharomyces cerevisiae external invertase isoforms. Food Chem. 2010 Jun 1;120(3):799-804. 
[16] Instituto Cubabo de Investigacones de los Derivados de la Caña de Azucar (ICIDCA). La industria de los derivados de la caña de azucar. Editorial. La Habana; 1986. 594 p.

[17] Cotopaxi G. Cantones [Internet]. 2015 [cited 2020 Jan 4]. Available from: https://www.cotopaxi.gob.ec/index.php/2015-09-20-01-15-34/secretaria-general/ itemlist/category/1cantones

[18] Holm S. A simple sequentially rejective multiple test Procedure. Board Found Scand J Stat. 1979;6(2):65-70.

[19] Friedman M. A comparison of alternative test of significance for the problem of $m$ rankings. Ann Math Stat. 1940;1:86-92.

[20] González Diaz YF, Hernández JG, Licea E. Empleo floculantes en la determinación del \% de pol en jugo de caña de azúcar. Tecnol Química [Internet]. 2016 [cited 2020 Jan 4];XXVI:21-5. Available from: http://www.redalyc.org/articulo.oa?id= 445543749003 